\title{
Engagement hacia un producto vs. hacia una marca: una escala para el contexto mexicano
}

\author{
Engagement towards a product vs. towards a brand: \\ A scale for the Mexican context \\ Jorge Vera-Martínez*, Sidney Ornelas-Sánchez \\ Escuela de Negocios. Tecnológico de Monterrey, México
}

Recibido el 14 de marzo de 2019; aceptado el 16 de noviembre de 2020

Disponible en Internet el: 23 de noviembre de 2020

\section{Resumen}

La atención hacia el concepto de engagement del consumidor se ha incrementado recientemente como un factor relevante en mercadotecnia. Esto ha motivado a realizar diversos estudios con el objetivo de lograr una mejor definición y medición de este constructo. El engagement se diferencia de conceptos como el involucramiento y la participación, siendo también un antecedente a variables como satisfacción y lealtad de marca. En este artículo, se propone y se pone a prueba la hipótesis de que existe una diferencia entre el grado de engagement (en sus tres dimensiones) hacia una categoría de producto y el grado de engagement hacia una marca particular dentro de esta categoría de producto. Para poner esta hipótesis a prueba, se presenta el proceso de adaptación y depuración de una escala para medir engagement en el contexto mexicano de consumo de café en taza. Se presentan los resultados de pruebas empíricas con las que verificó la confiabilidad y validez de la escala. Mediante pruebas de validez predictiva se corrobora al engagement como un antecedente de la satisfacción y de la lealtad de marca. Finalmente se presentan evidencias que confirman la hipótesis sobre la separación perceptual del engagement hacia la categoría del producto y hacia una marca.

\section{Código JEL: M31, M39, C18}

Palabras clave: Engagement del consumidor; Involucramiento del consumidor; Satisfacción del cliente; Lealtad hacia la marca; Engagement con la marca; Engagement con el producto

\footnotetext{
*Autor para correspondencia

Correo electrónico: jorge.vera@tec.mx (J. Vera-Martínez).

La revisión por pares es responsabilidad de la Universidad Nacional Autónoma de México. 


\title{
J. Vera-Martínez y S. Ornelas-Sánchez / Contaduría y Administración 66(3), 2021, 1-23 \\ http://dx.doi.org/10.22201/fca.24488410e.2021.1898
}

\begin{abstract}
The concept of consumer engagement has recently received increased attention as a relevant factor in marketing. This has led to several studies aiming to achieve better understanding and measurement for this construct. Engagement is distinct from other constructs such as involvement and participation; it also has been proposed as an antecedent to variables such as satisfaction and brand loyalty. In this article, a hypothesis about the difference between the level engagement (in its three dimensions) towards a product category and the level of engagement towards a particular brand in this category is discussed, tested and confirmed. In order to test the hypothesis, the process of adapting and debugging a scale to measure engagement in the Mexican context is also presented. The results of a series of empirical tests to verify the reliability and validity of the scale with Mexican consumers of grain coffee are presented as part of the process of refining the scale. Moreover, throughout predictive validity tests, engagement is confirmed as an antecedent of satisfaction and brand loyalty.
\end{abstract}

JEL Code: M31, M39, C18

Keywords: Consumer engagement; Consumer involvement; Customer satisfaction; Brand loyalty; Brand engagement; Product engagement

\section{Introducción}

El concepto de engagement en marketing ${ }^{1}$ hace referencia a una serie de ideas que incluyen la conexión, el apego emocional, la participación, el estado mental y el comportamiento social interactivo hacia un determinado objeto (Brodie, Hollebeek, Jurić, \& Ilić, 2011)the role of "customer engagement" (CE. El término engagement se utiliza en inglés a lo largo de este estudio dado que no existe una traducción al español que refleje totalmente el concepto, dado que posibles traducciones como involucramiento, compromiso, vinculación y participación, si bien están relacionados, no alcanzan a reflejar la esencia del constructo y podrían provocar confusión e imprecisión en su definición y medición. Por ejemplo, engagement e involvement son dos constructos que, si bien se relacionan, son distintos. Sin embargo, ambos se traducen al español como involucramiento. Las diferencias específicas con éste y otros conceptos se exponen en el marco conceptual más adelante.

Diversos investigadores han realizado distintos estudios en los últimos años con el objetivo de lograr una mejor definición y medición del engagement (Bowden, 2009; Brodie \& Hollebeek, 2011, a; Vivek, Beatty, \& Morgan, 2012). Para este trabajo, se realizó una búsqueda

\footnotetext{
${ }^{1}$ En este artículo se utiliza la palabra marketing en lugar de mercadotecnia. Esto debido a que es la forma generalizada en cómo se la llama a la disciplina en la mayoría de los países de habla hispana. El uso de la palabra "mercadotecnia" es una particularidad que sólo sucede en México.
} 
en literatura en español sobre este término para determinar si los diferentes vocablos que se han utilizado en este idioma han sido consistentes y distintos a otros conceptos relacionados. Como parte del interés por entender las aproximaciones hacia este constructo en un idioma distinto al inglés se encontraba también la necesidad de tener un instrumento de medición que acompañara su definición en el español. La revisión de literatura indica que hasta este momento no se ha publicado anteriormente una herramienta en idioma español que permita los indicadores asociados a las dimensiones del engagement. De tal manera, el presente trabajo tiene dos objetivos principales. Por un lado, analizar empíricamente si es posible diferenciar los niveles de engagement hacia el consumo de una categoría de producto (café en taza) y hacia el consumo de una marca dentro de esta misma categoría (Starbucks). En segundo lugar, presentar la adaptación (validez y confiabilidad) de una escala para medir engagement del consumidor en el contexto mexicano. Aunque en estudios anteriores se ha mostrado la diferencia de engagement entre distintos tipos de objetos, como se menciona más adelante, no se encontró en estudios previos que se pusiera a prueba esta diferencia en particular. Así, esto constituye una aportación original a la literatura por parte del presente trabajo. Esta diferencia es relevante ya que distinguir entre la percepción hacia productos y hacia sus distintas marcas es una forma de analizar el valor que una marca en particular puede agregar o no a un producto. Adicionalmente, se discuten las nociones asociadas al constructo y su posible utilidad tanto para investigaciones futuras, como para aplicaciones prácticas.

En este estudio, la categoría de producto -café en taza- se seleccionó con base en la identificación de una tendencia creciente de consumo que presenta nuevas dinámicas de mercado, desde su producción hasta su consumo, donde los consumidores han mostrado un interés incremental por este producto (Carvalho, Paiva, \& Vieira, 2016; Fischer \& Victor, 2014). En México, el café es un producto que ha sido parte de la cultura durante muchos años. Sin embargo, al ser uno de los principales países productores a nivel internacional, forma parte de un entorno que en años recientes se ha vuelto altamente competitivo tanto en los procesos productivos como en la forma en que se atiende la demanda y las necesidades y preferencias del consumidor (Morales Hernández, Mendez, Nolasco Ruíz, \& Cerón López, 2018). Adicionalmente, se ha registrado un incremento en la variedad de productos y marcas de café que buscan los consumidores, así como un alza en los precios que están dispuestos a pagar por él (Jones, 2016). Esta situación tiende a presionar a los miembros de la industria cafetalera en términos de que sean capaces de entender los cambios en la demanda para poder responder a ellos con innovaciones y adaptaciones. Adicionalmente, las tendencias mundiales tienden a empujar hacia el consumo de un café de mayor calidad, lo cual a su vez se relaciona con la aparición de un grupo de consumidores que han presentado un interés incremental por este producto (Fischer \& Victor, 2014; Hernandez, 2016). Este renovado interés y los cambios en la industria y el mercado evidencian la necesidad de poder medir adecuadamente el grado de 
engagement dentro de esta categoría, lo cual contribuiría a la comprensión de esta tendencia de sofisticación del mercado en un país productor como es México.

\section{Marco teórico}

El término engagement ha sido utilizado en diversas disciplinas que incluyen sociología, psicología, comportamiento organizacional, y más recientemente en marketing, abordándolo desde distintas perspectivas entre las que destacan el engagement de una persona hacia un determinado producto, marca u organización (Hollebeek, 2011, a). En el ámbito del marketing, si bien los campos de aplicación, los antecedentes y las consecuencias del engagement han sido diversos, los estudios principalmente se enfocan en un consumidor que presenta cierto nivel de engagement hacia un determinado objeto de este engagement como puede ser un producto, una marca u una organización (Dessart et al., 2016; Hollebeek, 2011, a). La revisión de literatura presentada a continuación incluye las principales definiciones del constructo, la distinción del mismo respecto a otros términos relacionados y el análisis de diversos instrumentos de medición.

Vivek et al (2012) definen el engagement del consumidor como la intensidad de la participación y la conexión de un individuo con la oferta de una organización entendiendo a los individuos como clientes actuales o potenciales. Brodie et al. (2011), por su parte, consideran que el engagement es un estado psicológico que ocurre tras experiencias interactivas del cliente con un agente $\mathrm{u}$ objeto focal que se manifiesta como un proceso iterativo y dinámico en una relación de co-creación de valor. Aquí, la co-creación de valor se refiere a la participación del consumidor en la creación de la oferta incluyendo actividades compartidas de invención, diseño y/o producción (Lusch \& Vargo, 2006). El engagement también ha sido considerado como un estado psicológico y como un proceso cognitivo que culmina en el apego conductual de un individuo hacia un objeto (Brodie et al., 2011). En marketing, este constructo representaría un estado mental y un proceso que puede llevar al consumidor a la lealtad hacia una marca, servicio, actividad o algún otro tipo de oferta empresarial (Vivek et al., 2012). Adicionalmente, Van Doorn et al. (2010) definen al engagement como la manifestación del comportamiento hacia una marca o empresa, más allá de la compra, que resulta de ciertas motivaciones.

El concepto de engagement del consumidor ha tomado especial relevancia en ámbitos empresariales tras identificarse como un elemento que puede ser relevante para el logro de objetivos de negocio que incluyen un mejor desempeño, un incremento en ventas, y/o una ventaja competitiva (Brodie et al., 2011). Así, existe evidencia de que un engagement superior, que puede generar una conexión más cercana entre el cliente y la marca, ha tenido un efecto significativo en comportamientos como la satisfacción del consumidor y su lealtad hacia la marca (Hollebeek, 2011, b). Esto ha motivado, de forma paralela, un incremento en el aná- 
lisis de este concepto en trabajos académicos donde se han estudiado sus bases teóricas, sus antecedentes, y sus aplicaciones, así como su utilidad como predictor de comportamientos. El engagement del cliente resulta relevante para el ámbito del marketing debido a que se refiere a una manifestación del comportamiento altamente relacionado con las transacciones comerciales (Verhoef, Reinartz, \& Krafft, 2010). En las últimas dos décadas la implementación de estrategias para manejar las relaciones cliente-empresa/marca/producto ha sido una tendencia constante. Es importante recalcar que, si bien el marketing de relaciones también ha recibido gran atención académica, este último tiende a dirigirse hacia las acciones de la empresa, mientras que el engagement busca entender desde el punto de vista del cliente (Vivek et al., 2012).

Vivek et al. (2012) encontraron que el engagement puede presentarse hacia una oferta de marcas, productos y servicios o hacia actividades como comprar y participar en seminarios. Por su parte, Brodie et al. (2011) realizaron una revisión del concepto en la literatura académica y sus definiciones, encontrando que cada una de ellas adopta un enfoque ya sea cognitivo, emocional o de comportamiento. El enfoque cognitivo se centra en la manera en que el individuo se relaciona con el aprendizaje del objeto. El afectivo se refiere al nivel de apego con el mismo. El de comportamiento se centra en las interacciones del individuo con el objeto. Este último es el que mayormente recibe la atención de la investigación en marketing ya que los comportamientos incluyen variables que generalmente son consideradas meta para los practicantes en esta área como son: la lealtad a la marca (en su componente actitudinal y de comportamiento a través de la compra) y el compromiso con la misma (Vivek, Beatty, Dalela, \& Morgan, 2014).

El engagement se ha relacionado con diversos constructos ampliamente estudiados en la investigación de marketing. Es importante distinguir y explorar la relación que estos otros conceptos guardan con el engagement. Uno de ellos es el involucramiento del consumidor, el cual se define como la relevancia percibida de un objeto basado en necesidades, intereses y valores inherentes (Zaichkowsky, 1985). El involucramiento es un constructo que indica un estado mental de relevancia personal pero no se identifica como un comportamiento, por lo que se ubica como un antecedente al engagement a través de una asociación positiva entre el nivel de involucramiento y la intensidad del engagement con un determinado objeto o actividad (Vivek et al., 2012). Mientras más involucrado se encuentre el sujeto, mayor será la intensidad del engagement con un determinado objeto o actividad. Así, el involucramiento ha sido típicamente definido como un estado interno de excitación e importancia que un consumidor puede tener por una categoría de producto en relación a sus intereses personales y sistema de valores, lo cual motiva a la búsqueda de información (Bowden, 2009) y en algunos casos a la generación de engagement (Hollebeek, 2011, a). El engagement, por su parte, es crucial para entender al consumidor y el proceso que lo lleva a cierto comportamiento (Pansari \& Kumar, 
2017) y se define como la intensidad de la conexión de un consumidor con un producto, servicio o actividad (Vivek et al., 2012) y como un estado psicológico que resulta de la interacción entre un consumidor un objeto focal (marca, empresa o actividad (Brodie et al., 2011). Por lo tanto, el engagement puede resultar más complejo que el involucramiento, el cual incluye componentes actitudinales, así como elementos de comportamiento, que se extienden hacia la relación interactiva con la marca, más allá de un simple estado interno del consumidor.

Otro de los constructos relacionados con el engagement es la participación en la co-creación de valor, la cual es definida como el grado en el que un consumidor se involucra en el proceso de producción o entrega del servicio (Dabholkar, 1990). Esta interacción puede producir diferentes niveles de entusiasmo y por lo tanto, mayor engagement (Vivek et al., 2012). Por esta razón, el involucramiento y la participación se identifican como antecedentes al engagement. Por otro lado, el valor, la confianza, el compromiso afectivo, la comunicación boca a boca, la lealtad y el involucramiento con una comunidad de marca son considerados consecuencias del mismo (Vivek et al., 2012). El compromiso y la lealtad son distintos debido a que el compromiso se relaciona más directamente con una actitud. La lealtad, por su parte, si bien contiene un elemento actitudinal, se puede relacionar más con un comportamiento. Así, el engagement incluye tanto aspectos cognoscitivos como elementos conductuales (Bowden, 2009).

Además de buscar una mejor definición de engagement, ha sido necesario identificar si existen instrumentos que puedan medirlo adecuadamente. Así como la traducción del término mismo genera confusión al existir términos hispanos utilizados indistintamente, al querer utilizar el constructo empíricamente, no ha sido posible encontrar una herramienta válida y confiable desarrollada para países de habla hispana. Por esta razón se llevó a cabo una revisión de las escalas disponibles en idioma inglés, para después elegir una que permitiera realizar su traducción y validación para un contexto hispano hablante y a su vez permitiera aplicarse a distintos objetos de engagement.

\section{Medición del engagement}

Diversos investigadores han propuesto distintas definiciones y herramientas de medición para el engagement. En algunos casos, este constructo se ha planteado como uno de tipo unidimensional (Balsano, 2005; Catteeuw, Flynn, \& Vonderhorst, 2007; Guthrie \& Cox, 2001; van Doorn et al., 2010). En otros casos, se ha propuesto como uno de tipo multidimensional (Bowden, 2009; Higgins \& Scholer, 2009; Mollen \& Wilson, 2010; Vivek et al., 2012). El enfoque multidimensional ha sido predominante en el área de marketing, mientras que las aproximaciones unidimensionales tienden a estar ligadas con ámbitos que incluyen el comportamiento organizacional, la educación y las ciencias políticas, entre otros. Así, para 
propósitos del presente artículo se utiliza un enfoque tridimensional propuesto por Vivek et al. (2014) que se desarrolla más adelante.

La relevancia de la medición y el entendimiento del engagement del consumidor reside en las consecuencias que tiene para los clientes y para las empresas. Según el modelo propuesto por Van Doorn et al. (2010), los clientes podrían manifestar distintos grados de engagement a una marca como respuesta a diferentes estrategias, y con esto, podrían contribuir a la creación de valor de manera mucho más activa. En los programas de lealtad, por ejemplo, donde se muestran comportamientos de engagement, el cliente recibe una recompensa financiera. Para la empresa, un engagement más elevado por parte del cliente hacia su marca puede repercutir en beneficios financieros al generar una mayor comunicación boca a boca o un mayor reconocimiento de marca (van Doorn et al., 2010).

Se han realizado diversos estudios con el objetivo de crear instrumentos que puedan medir el engagement de una manera adecuada (Dessart, Veloutsou, \& Morgan-Thomas, 2016; Hollebeek, Glynn \& Brodie, 2014; Sprott, Czellar, \& Spangenberg, 2009; Vivek et al., 2014). Sin embargo, muchas de estas escalas están enfocadas en ciertos objetos específicos hacia los cuales se puede generar engagement. Algunos, por ejemplo, se enfocan en el engagement hacia las comunidades virtuales (Baldus, Voorhees, \& Calantone, 2015; Dessart et al., 2016); otros, hacia la industria del turismo (So, King, \& Sparks, 2014). Además de su difícil aplicabilidad en distintos contextos, todos estos instrumentos han sido desarrollados en idioma inglés y su traducción no ha sido validada al idioma español. Por esta razón, este trabajo busca responder a esta necesidad, tomando como base una escala que pueda ser aplicada en distintos contextos y además considere los componentes actitudinales y conductuales que han sido consistentes en los diversos análisis del engagement.

La escala presentada por Vivek et al. (2014), la cual ha sido seleccionada para este estudio, destaca por su naturaleza de generalización. Esta escala, la cual aborda al engagement de manera multidimensional, consta de 10 reactivos englobados en tres dimensiones. Dichas dimensiones son consistentes con hallazgos previos en la literatura de mercadotecnia (Calder, Malthouse, \& Schaedel, 2009; Gambetti, Graffigna, \& Biraghi, 2012; Haven, 2007; L. L. D. Hollebeek et al., 2014). Así, la propuesta tridimensional de Vivek et al. está conformada por las siguientes dimensiones: 1) Atención consciente: grado de interés que la persona tiene o desea tener en interactuar con el foco de su engagement; 2) participación entusiasta: reacciones y emociones relacionadas con el uso o la interacción del foco de su engagement; 3 ) conexión social: incremento de la interacción basada en la inclusión de terceros con el foco del engagement lo que indicaría una acción especial recíproca en la presencia de otros. La característica de generalización se debe a que este instrumento es una escala multi-objeto, donde el diseño de los reactivos permite que se adapten hacia distintos focos de engagement (producto, marca, actividad, etcétera). Las tres dimensiones, que ya fueron mencionadas, se 
refieren a los componentes actitudinales y conductuales del engagement. Así, el hecho de que el instrumento considere la posibilidad de adaptarse a distintos focos de engagement permite hacer comparaciones de niveles entre objetos de diferente naturaleza.

Un objetivo crucial de este estudio es el analizar empíricamente la diferencia entre el engagement hacia el consumo de una categoría de producto (café en taza) y hacia el consumo de una marca dentro de esa categoría (Starbucks). Para lograr este objetivo, se plantean las siguientes hipótesis:

H1 El nivel de engagement hacia una categoría de producto (en este caso, café en taza) es distinto al que se presenta hacia una marca dentro de la misma categoría de producto (en este caso, Starbucks).

H2 Las tres dimensiones de engagement presentan diferentes niveles cuando se miden para una categoría de producto (en este caso, café en taza) que cuando se analiza a nivel de marca dentro de la misma categoría de producto (en este caso, Starbucks). Esto da pie a las siguientes sub-hipótesis:

-H2a El nivel de atención consciente de un consumidor hacia una categoría de producto es distinto al que presenta hacia una marca dentro de esa misma categoría.

- H2b El nivel de participación entusiasta de un consumidor hacia una categoría de producto es distinto al que presenta hacia una marca dentro de esa misma categoría.

-H2c El nivel de conexión social de un consumidor hacia una categoría de producto es distinto al que presenta hacia una marca dentro de esa misma categoría.

Estas hipótesis podrán ser aceptadas o rechazadas tras haber realizado el proceso de adaptación y validación del instrumento y a su vez, se contribuye al establecimiento de una validez externa, al comprobar su capacidad de generalización en contextos distintos. El proceso de construcción de la escala incluye una fase inicial cualitativa para adaptar y definir los reactivos, una siguiente fase para validar dichos reactivos con una base teórica, una fase más donde se realizaron una serie de pruebas de confiabilidad y de validez por convergencia-discriminación para la purificación de la escala, y finalmente, una aplicación para poner a prueba su validez predictiva.

\section{Metodología}

Para realizar la adaptación al español, se llevó a cabo un análisis de la escritura de los reactivos de la escala de Vivek et al. (2014). Este análisis incluyó una revisión por parte de cinco expertos en el campo de marketing. Estos expertos fueron seleccionados por ser bilingües y 
por tener una trayectoria destacada en el área de marketing. Ellos presentaron sugerencias valiosas para los términos utilizados en algunos reactivos. Desde esta primera etapa, se determinó utilizar como objetos de engagement una categoría de producto (café en taza) y una marca (Starbucks).

\section{Interpretación y selección de reactivos}

Se utilizó como base el conjunto inicial de 40 reactivos utilizados por Vivek et al. (2014). Después del proceso de depuración, cada una de las 3 dimensiones quedaron con 6 reactivos para una escala final de 18 reactivos en total. Este proceso de depuración de reactivos se llevó a cabo considerando aquellas afirmaciones cuyo sentido, una vez traducidas al español, generan menos ambigüedades y un mejor entendimiento. Se realizó una adaptación al español enriquecida con aportaciones de tres expertos, cuyo perfil ha sido previamente descrito, para elegir los términos más adecuados. Se realizaron las pruebas pertinentes a los dieciocho reactivos bajo la premisa de que la escala final constaría de un número menor de reactivos debido al proceso normal de depuración de una escala. Estos 18 reactivos se muestran en la Tabla 1.

Como se puede ver en la Tabla 1, los reactivos del instrumento están diseñados de tal forma que permiten adaptarse a diferentes objetos de engagement, en este caso la categoría del producto: café en taza, y una marca dentro de la categoría: Starbucks. Esta marca se seleccionó por ser una con un alto nivel de reconocimiento para los sujetos que participaron en las pruebas. Dada la variedad de productos ofrecidos por esta marca y para poder hacer una comparación más clara con la categoría de producto, se hizo la aclaración, al momento de aplicar el instrumento, de considerar solamente el consumo de un café tipo americano, para evitar sesgos. 
Tabla 1

Escala de engagement del consumidor para el contexto mexicano. Reactivos seleccionados para la primera prueba de campo

\begin{tabular}{|c|c|}
\hline Dimensión & Reactivos \\
\hline Atención consciente & $\begin{array}{l}\text { 1. Cualquier asunto relacionado con _ llama mi atención. } \\
\text { 2. Me gusta aprender sobre } \\
\text { 3. Pongo mucha atención a todo lo relacionado con } \\
\text { 4. Me mantengo al tanto de novedades relacionadas con } \\
\text { 5. __apta mi atención a menudo. } \\
\text { 6. Siempre hay alguna novedad relacionada con }\end{array}$ \\
\hline Participación entusiasta & $\begin{array}{l}\text { 1. Dedico mucho de mi tiempo personal a __. } \\
\text { 2. Me considero apasionado por } \\
\text { 3. Mis días no serían iguales sin } \\
\text { 4. _. es una parte importante en mi vida. } \\
\text { 5. Busco hacer espacios en mi agenda para dedicar a } \\
\text { 6. Pierdo la noción del tiempo cuando }\end{array}$ \\
\hline Conexión social & $\begin{array}{l}\text { 1. Me encanta _on mis amigos. } \\
\text { 2. Disfruto más cuando estoy acompañado. } \\
\text { 3. _es más divertido cuando hay más gente conmigo que también lo hace. } \\
\text { 4. Me gusta que los demás vean qué tanto me gusta } \\
\text { 5. Busco interactuar con personas a quienes también les gusta __ } \\
\text { 6. Hablo mucho con mis amigos sobre }\end{array}$ \\
\hline
\end{tabular}

\section{Selección final de reactivos}

Se realizó una primera prueba donde una muestra de $n=60$ sujetos respondieron a un cuestionario que incluía los 18 reactivos. El tamaño de muestra es consistente con las recomendaciones para desarrollo inicial de escalas (Johanson \& Brooks, 2010). Los objetivos de esta primera prueba fueron: (1) identificar posibles confusiones de significado para los sujetos; (2) discriminar si las dimensiones propuestas por Vivek et al. se replican en el contexto mexicano (esto mediante un análisis factorial confirmatorio); (3) identificar redundancias. El instrumento se aplicó a través de un cuestionario en línea, el cual se envió a los n=60 sujetos. Para la participación de estos sujetos, se verificó que cumplieran con ciertas características clave para el estudio: (1) ser consumidores de café en taza, (2) ser mayores de edad y (3) conocer la marca Starbucks. Otra característica importante de la muestra es que los sujetos se seleccionaron a través de un muestreo no-aleatorio por juicio, pero, verificando cierta homogeneidad. De tal forma, los respondientes fueron seleccionados dentro de un nivel socioeconómico medio y medio-alto. Este segmento tiende a ser consistente con el mercado meta de la marca seleccionada (Starbucks). Los reactivos fueron asociados a una escala de Likert de 5 puntos que va de "totalmente de acuerdo" a "totalmente en desacuerdo". 
La Tabla 2 muestra los resultados de la prueba de validez por convergencia y discriminación mediante un análisis factorial confirmatorio (con rotación) para el nivel de producto (café en taza). De forma similar, la Tabla 3 muestra estos resultados al nivel de marca (Starbucks). Aunque, como se irá viendo, en este trabajo se utilizan métodos estadísticos de tipo paramétrico (ej. análisis factorial, regresión), y las variables utilizadas son de tipo ordinal/ discreto, existen evidencias de que los métodos paramétricos lineales consistentemente generan resultados confiables a pesar de que existan ciertas violaciones a los supuestos de lo paramétrico como pueden ser la normalidad de los datos y el nivel de medición (Norman, 2010). Estos análisis tienden a confirmar la tridimensionalidad propuesta por el instrumento original, donde los reactivos y sus cargas factoriales se agrupan de manera congruente con la dimensión a la que originalmente pertenecen.

Tabla 2

Primera prueba de campo. Análisis factorial confirmatorio, nivel producto

\begin{tabular}{|c|c|c|c|c|}
\hline \multirow[b]{2}{*}{ Reactivos } & \multirow[b]{2}{*}{ Dimensión } & \multicolumn{3}{|c|}{ Componentes } \\
\hline & & 1 & 2 & 3 \\
\hline Mis días no serían iguales sin tomar café. & PE3 & 0.899 & & \\
\hline Tomar café es una parte importante en mi vida. & PE4 & 0.884 & & \\
\hline Busco hacer espacios en mi agenda para dedicar a tomar café. & PE5 & 0.82 & & \\
\hline Me gusta que los demás vean qué tanto me gusta tomar café. & CS4 & 0.781 & & \\
\hline Dedico mucho de mi tiempo personal a tomar café. & PE1 & 0.757 & & \\
\hline Hablo mucho con mis amigos sobre asuntos relacionados a tomar café. & CS6 & 0.711 & & \\
\hline Pierdo la noción del tiempo cuando tomo café. & PE6 & 0.616 & & \\
\hline Busco interactuar con personas a quienes también les gusta tomar café. & CS5 & 0.614 & & \\
\hline Me considero apasionado por tomar café. & PE2 & & & \\
\hline Disfruto más tomar café cuando estoy acompañado. & $\mathrm{CS} 2$ & & 0.926 & \\
\hline $\begin{array}{l}\text { Tomar café es más divertido cuando hay más gente conmigo que } \\
\text { también. }\end{array}$ & $\mathrm{CS} 3$ & & 0.878 & \\
\hline Me encanta tomar café con mis amigos. & CS1 & & 0.623 & \\
\hline Me gusta aprender cosas relacionadas con tomar café. & $\mathrm{AC} 2$ & & & 0.944 \\
\hline Me mantengo al tanto de novedades relacionadas con tomar café. & $\mathrm{AC} 4$ & & & 0.93 \\
\hline Pongo mucha atención a todo lo relacionado con tomar café. & AC3 & & & 0.924 \\
\hline Siempre hay alguna novedad sobre cómo tomar café. & AC6 & & & 0.684 \\
\hline Cualquier asunto relacionado con tomar café llama mi atención & $\mathrm{AC} 1$ & & & 0.654 \\
\hline Tomar café capta mi atención a menudo. & AC5 & & & 0.630 \\
\hline
\end{tabular}

Nota: Análisis factorial confirmatorio con rotación oblimin usando componentes principales. Escala 1 (totalmente en desacuerdo) a 5 (totalmente de acuerdo). AC= Atención consciente, $\mathrm{PE}=$ Participación entusiasta, $\mathrm{CS}=$ Conexión social. Se omiten cargas factoriales por debajo de 0.5

Fuente: elaboración propia 
Tabla 3

Primera prueba de campo. Análisis factorial Confirmatorio, nivel marca

\begin{tabular}{lccc}
\hline \multicolumn{1}{c}{ Reactivos } & \multicolumn{3}{c}{ Componentes } \\
& Dimensión & 1 & 2 \\
\hline Me gusta aprender sobre Starbucks. & AC2 & 0.962 & \\
Pongo mucha atención a todo lo relacionado con Starbucks. & AC3 & 0.913 & \\
Starbucks capta mi atención a menudo. & AC5 & 0.834 & \\
Me mantengo al tanto de novedades relacionadas con Starbucks. & AC4 & 0.831 \\
Cualquier asunto relacionado con Starbucks llama mi atención. & AC1 & 0.830 & \\
Siempre hay alguna novedad relacionada con Starbucks. & AC6 & 0.674 & \\
Starbucks es una parte importante en mi vida. & PE4 & & -0.972 \\
Mis días no serían iguales sin ir a Starbucks. & PE3 & & -0.926 \\
Busco hacer espacios en mi agenda para ir a Starbucks. & PE5 & & -0.833 \\
Me gusta que los demás vean qué tanto me gusta Starbucks. & CS4 & & -0.783 \\
Dedico mucho de mi tiempo personal a Starbucks. & PE1 & & -0.779 \\
Pierdo la noción del tiempo cuando voy a Starbucks. & PE6 & & -0.757 \\
Hablo mucho con mis amigos sobre Starbucks. & CS6 & & -0.731 \\
Busco interactuar con personas a quien también les guste & & & \\
Starbucks. & CS5 & & \\
Me considero apasionado por Starbucks. & PE2 & & \\
Disfruto más Starbucks cuando estoy acompañado. & CS2 & & \\
Starbucks es más divertido cuando hay más gente conmigo que... & CS3 & \\
Me encanta ir a Starbucks con mis amigos. & CS1 & & \\
\hline
\end{tabular}

Nota: Análisis factorial confirmatorio con rotación oblimin usando componentes principales. Escala 1 (totalmente en desacuerdo) a 5 (totalmente de acuerdo). $\mathrm{AC}=$ Atención consciente, $\mathrm{PE}=$ Participación entusiasta, $\mathrm{CS}=$ Conexión social. Se omiten cargas factoriales por debajo de 0.5

Fuente: elaboración propia 


\section{Segunda prueba y validación de escala}

Considerando los resultados del análisis factorial, se eliminaron los reactivos con las cargas factoriales más bajas y aquellos que no contribuían a la explicación del factor correspondiente a la dimensión que la revisión de literatura indicaba. Para mantener el balance del instrumento, se agregaron dos nuevos reactivos a la dimensión de conexión social. La Tabla 4 muestra los reactivos seleccionados para la segunda prueba de campo. Los reactivos seleccionados se utilizaron para elaborar un nuevo instrumento, el cual se aplicó bajo las mismas condiciones de la primera prueba de campo, a 60 personas con las mismas características descritas anteriormente.

Tabla 4

Escala de engagement del consumidor para el contexto mexicano

\begin{tabular}{|c|c|}
\hline Dimensión & Reactivos \\
\hline \multirow{4}{*}{ Atención consciente } & 1. Me gusta aprender sobre \\
\hline & 2. Pongo mucha atención a todo lo relacionado con \\
\hline & 3. Me mantengo al tanto de novedades relacionadas con \\
\hline & 4.___ capta mi atención a menudo. \\
\hline \multirow{4}{*}{$\begin{array}{c}\text { Participación } \\
\text { entusiasta }\end{array}$} & 1. Dedico mucho de mi tiempo personal a \\
\hline & 2. Mis días no serían iguales sin \\
\hline & 3.___ es una parte importante en mi vida. \\
\hline & 4. Busco hacer espacios en mi agenda para dedicar a \\
\hline \multirow{4}{*}{ Conexión social } & 1. Disfruto más___ cuando estoy acompañado. \\
\hline & 2. __ es más divertido cuando hay más gente conmigo que también lo hace. \\
\hline & 3. es algo que comparto con mis amigos. \\
\hline & 4. es una buena manera de convivir con familia y amigos. \\
\hline
\end{tabular}

Fuente: elaboración propia

Una vez aplicada la segunda prueba de campo, se realizó nuevamente un análisis de validez por convergencia y discriminación mediante un análisis factorial con rotación. Estos resultados se muestran en la Tabla 5 para el nivel producto, y en la Tabla 6 para el nivel marca. 
Tabla 5

Segunda prueba de campo. Análisis factorial Confirmatorio, nivel producto

Componentes

\begin{tabular}{|c|c|c|c|c|}
\hline Reactivos & Dimensión & 1 & 2 & 3 \\
\hline Pongo mucha atención a todo lo relacionado con tomar café. & P_AC2 & 0.925 & & \\
\hline Me gusta aprender sobre café. & P_AC1 & 0.901 & & \\
\hline El café capta mi atención a menudo. & P_AC4 & 0.861 & & \\
\hline $\begin{array}{l}\text { Me mantengo al tanto de las novedades relacionadas con el } \\
\text { café. }\end{array}$ & P_AC3 & 0.799 & & \\
\hline Disfruto más tomar café cuando estoy acompañado. & P_CS1 & & 0.966 & \\
\hline $\begin{array}{l}\text { Tomar café es más divertido cuando estoy con gente que } \\
\text { también... }\end{array}$ & P_CS2 & & 0.944 & \\
\hline Tomar café es algo que comparto con mis amigos. & P_CS3 & & 0.774 & \\
\hline $\begin{array}{l}\text { Tomar café es una buena manera de convivir con familia y } \\
\text { amigos. }\end{array}$ & P_CS4 & & 0.691 & \\
\hline Mis días no serían iguales sin tomar café. & P_PE2 & & & 0.989 \\
\hline Tomar café es una parte importante en mi vida. & P_PE3 & & & 0.871 \\
\hline Busco hacer espacios en mi agenda para dedicar a tomar café. & P_PE4 & & & 0.711 \\
\hline Dedico mucho de mi tiempo personal a tomar café. & P_PE1 & & & 0.612 \\
\hline
\end{tabular}

Nota: Análisis factorial confirmatorio con rotación oblimin. Escala 1 (totalmente en desacuerdo) a 5 (totalmente de acuerdo). AC=Atención consciente, $\mathrm{PE}=$ Participación entusiasta, $\mathrm{CS}=$ Conexión social. Se omiten cargas factoriales por debajo de 0.5

Fuente: elaboración propia 
Tabla 6

Segunda prueba de campo. Análisis factorial Confirmatorio, nivel marca

\begin{tabular}{|c|c|c|c|c|}
\hline \multirow[b]{2}{*}{ Reactivos } & \multirow[b]{2}{*}{ Dimensión } & \multicolumn{3}{|c|}{ Componentes } \\
\hline & & 1 & 2 & 3 \\
\hline Me gusta aprender sobre Starbucks. & M_AC1 & 0.855 & & \\
\hline Pongo mucha atención a todo lo relacionado con Starbucks. & M_AC2 & 0.845 & & \\
\hline Starbucks capta mi atención a menudo. & M_AC4 & 0.814 & & \\
\hline Me mantengo al tanto de las novedades relacionadas con Starbucks. & M_AC3 & 0.805 & & \\
\hline Disfruto más Starbucks cuando estoy acompañado. & M_CS1 & & 0.924 & \\
\hline Starbucks es más divertido cuando estoy con gente que también le & & & & \\
\hline gusta... & M_CS2 & & 0.869 & \\
\hline Starbucks es algo que comparto con mis amigos. & M_CS3 & & 0.764 & \\
\hline Starbucks es una buena manera de convivir con familia y amigos. & M_CS4 & & 0.578 & \\
\hline Starbucks es una parte importante en mi vida. & M_PE3 & & & 0.943 \\
\hline Busco hacer espacios en mi agenda para dedicar a Starbucks. & M_PE4 & & & 0.924 \\
\hline Mis días no serían iguales sin ir a Starbucks. & M_PE2 & & & 0.898 \\
\hline Dedico mucho de mi tiempo personal a Starbucks. & M_PE1 & & & 0.531 \\
\hline
\end{tabular}

Nota: Análisis factorial confirmatorio con rotación oblimin, forzada a 3 factores. Escala 1 (totalmente en desacuerdo) a 5 (totalmente de acuerdo). AC= Atención consciente, $\mathrm{PE}=$ Participación entusiasta, $\mathrm{CS}=$ Conexión social. Se omiten cargas factoriales por debajo de 0.5

Fuente: elaboración propia

A través del análisis factorial fue posible establecer la validez del instrumento al verificarse la convergencia de los reactivos en tres factores en ambos niveles (producto y marca): atención consciente, participación entusiasta y conexión social. En cuanto al ajuste del modelo, la prueba KMO arrojó un indicador de .809 para nivel producto y .861 en el nivel marca. Así mismo, los modelos para ambos niveles resultaron significativos en la prueba de Bartlett. Esto tiende a indicar que el modelo explica adecuadamente a las variables observadas. Por otro lado, la consistencia interna se muestra a través de los coeficientes alfa de Cronbach (Tabla 7), los cuales muestran niveles de confiabilidad que se pueden considerar altamente aceptables (Gliem \& Gliem, 2003). 
Tabla 7

Segunda prueba de campo. Análisis de consistencia interna: Alfa de Cronbach

\begin{tabular}{lccc}
\hline & & \multicolumn{2}{c}{ Alfa de Cronbach } \\
Dimensión & Número de reactivos & Producto: Café & Marca: Starbucks \\
\hline Atención Consciente & 4 & 0.927 & 0.929 \\
Pariticipación Entusiasta & 4 & 0.916 & 0.938 \\
Conexión Social & 4 & 0.899 & 0.904 \\
Escala Engagement & 12 & 0.918 & 0.938 \\
\hline
\end{tabular}

Fuente: elaboración propia

\section{Validez predictiva}

Dado que se ha establecido previamente que tiende a existir una asociación entre el engagement hacia la marca con la lealtad de marca, así como con la satisfacción del cliente (Hollebeek, 2011, b), se incluyeron dentro de esta segunda prueba tres reactivos para la medición de indicadores de cada uno de estos dos constructos (Oliver, 1999). Así, se llevaron a cabo pruebas de validez predictiva de la escala. Los reactivos utilizados y los resultados del análisis factorial para cada uno (lealtad de marca y satisfacción del cliente) se muestran en la Tabla 8.

Tabla 8

Análisis factorial de variables dependientes: Lealtad y Satisfacción

\begin{tabular}{|c|c|c|c|}
\hline & & $\begin{array}{c}\text { \% Varianza } \\
\text { explicada }\end{array}$ & $\begin{array}{c}\text { Alfa de } \\
\text { Cronbach }\end{array}$ \\
\hline \multirow{3}{*}{ LEALTAD } & La próxima vez que compre café, será Starbucks. & \multirow{3}{*}{78.7} & \multirow{3}{*}{0.85} \\
\hline & Recomendaría el café de Starbucks a un amigo. & & \\
\hline & Volvería a tomar café de Starbucks & & \\
\hline \multirow{3}{*}{ SATISFACCIÓN } & El café de Starbucks cumple con mis expectativas. & \multirow{3}{*}{91.06} & \multirow{3}{*}{0.95} \\
\hline & Me gusta el café de Starbucks. & & \\
\hline & El café de Starbucks me da lo que me gusta de un café. & & \\
\hline
\end{tabular}

Método de extracción: Componentes principales

Fuente: elaboración propia 
Los reactivos para lealtad de marca y para satisfacción fueron sometidos en sendos análisis factoriales para corroborar su validez de convergencia. Posteriormente, se utilizaron los puntajes factoriales de cada uno como variables (latentes) dependientes en las pruebas de validez predictiva. De tal forma, con estas dos variables latentes, se elaboraron dos modelos de regresión donde las variables independientes fueron los puntajes factoriales de los componentes en los que convergieron los reactivos de engagement. A diferencia de los análisis factoriales anteriores, estos puntajes factoriales se obtuvieron con rotación varimax. Esta, es un tipo de rotación de tipo ortogonal para evitar problemas de multicolinealidad de las variables independientes en los modelos de causalidad (como los de regresión). Los resultados de los modelos de regresión se muestran en la Tabla 9.

Tabla 9

Modelos de regresión. Efectos de las dimensiones en la lealtad

\begin{tabular}{lcccccc}
\hline & & \multicolumn{5}{c}{ Coeficientes } \\
Variable dependiente & Variable independiente & estandarizados & $R$ & $R^{\wedge} 2$ & $F$ \\
\hline \multirow{2}{*}{ Modelo 1 } & \multirow{2}{*}{ LEALTAD } & Atención Consciente & $0.589^{* *}$ & 0.77 & 0.59 & $26.9^{* *}$ \\
& & Participación Entusiasta & $0.28^{* *}$ & & & \\
& & Conexión Social & $0.407^{* *}$ & & & \\
\hline \multirow{2}{*}{ Modelo 2 } & \multirow{2}{*}{ SATISFACCIÓN } & Atención Consciente & $0.483^{* *}$ & 0.65 & 0.43 & $14.1^{* *}$ \\
& & Participación Entusiasta & 0.149 & & & \\
& & Conexión Social & $0.418^{* *}$ & & & \\
\hline
\end{tabular}

$\mathrm{n}=60 * *$ Significancia inferior a .01

Fuente: elaboración propia

El modelo obtenido para lealtad de marca confirma los resultados obtenidos en estudios previos donde se propone a este constructo como una consecuencia del engagement. La lealtad de marca ha sido propuesta como una consecuencia de compra repetida y otros comportamientos derivados de un proceso de engagement hacia una determinada marca (Bowden, 2009). El modelo obtenido sostiene la relevancia de las tres dimensiones propuestas (variables independientes) para explicar la lealtad hacia la marca Starbucks al tener coeficientes significativos. En el caso del modelo para la variable satisfacción, la dimensión de participación entusiasta (variable independiente) resultó ser no significativa. Esto puede sugerir la necesidad de revisar los modelos en los que se vincula el engagement con la satisfacción, particularmente en la dimensión de participación entusiasta. De igual manera, podría explorarse la relevancia de cada una de las dimensiones en categorías de producto diferentes ya que, si bien se ha corroborado que se conservan las tres dimensiones en contextos diferente, su relevancia puede tender a diferir en cada uno de ellos. 


\section{Contrastación de hipótesis: diferenciando entre producto y marca}

Poner a prueba la escala en un contexto de categoría de producto y en un contexto de marca sirve para argumentar la validez multi-objeto del instrumento. En el presente estudio, al analizar el constructo en dos niveles (producto y marca), se busca además de confirmar la validez de la escala en distintos contextos, proponer trabajos de investigación que profundicen sobre las implicaciones de distintos grados de engagement en ambos niveles. Resulta interesante encontrar que un sujeto pueda presentar distintos grados de engagement hacia una categoría de producto y hacia una marca dentro de esta categoría, tal como lo demuestran las diferencias significativas de las medias entre ambos niveles probados (producto y marca) para cada uno de los reactivos como se observa en la Tabla 10. En estas pruebas, en las cuales se utiliza valor promedio del grado de acuerdo para cada reactivo, se evidencia que un mismo individuo puede presentar un grado de engagement más alto para una categoría que para una marca en específico. Esta dualidad de observar distintos niveles de engagement entre dos objetos distintos, pero relacionados, ha sido estudiada previamente donde se encontraron diferentes niveles de engagement entre una marca y una comunidad de marca en un contexto de internet (Dessart et al., 2016).

La dualidad descrita previamente resalta la importancia de contar con escalas que reconozcan y analicen estas variaciones en el engagement para distintos objetos (Dessart et al., 2016). Si bien el constructo se define como multidimensional, el hecho de que los valores de cada una de estas dimensiones pueden ser distinta para un mismo consumidor en distintos niveles de análisis, presenta oportunidades relevantes para futuras investigaciones. Por ejemplo, el entender cómo el engagement hacia diferentes objetos puede tener efecto en comportamientos como la elección y preferencia de marca y como cada una de las dimensiones tiene un efecto distinto para estos comportamientos, puede generar estrategias de mercadotecnia mejor enfocadas y más efectivas. Los hallazgos del presente estudio plantean también la posibilidad de establecer un indicador de comparación entre marcas, tomando como centro de referencia el nivel de engagement para la categoría de producto.

Para continuar con el análisis de las diferencias estadísticas entre el engagement hacia la categoría de producto y hacia la marca, adicionalmente se realizó un análisis factorial con rotación varimax forzado a 2 factores. Se obtiene un valor de 0.78 en el coeficiente KMO y un resultado significativo en la prueba de esfericidad de Bartlett. Estos resultados indican que los dos factores obtenidos representan de forma adecuada a las variables observadas introducidas en el análisis. En la Tabla 11, se puede ver como los reactivos del engagement hacia la categoría de producto obtienen cargas factoriales altas en un factor distinto al factor en el cual los reactivos del engagement hacia la marca obtienen cargas altas. Dado que la rotación varimax asegura la correlación cero entre los factores resultantes, se sustenta con claridad la 
separación estadística entre los dos tipos de indicadores. Así, con los datos mostrados en la Tabla 10 y en la Tabla 11, se ofrece evidencia de la separación del engagement producto/marca tanto con las variables observadas como con variables latentes. De tal forma, los resultados del estudio proporcionan evidencia que tiende a sustentar las hipótesis planteadas tanto para la separación del engagement total entre la categoría de producto y una marca dentro de esa misma categoría (H1), como la separación de cada una de las dimensiones del engagement (atención consciente, participación entusiasta conexión social) entre la categoría de producto y una marca dentro de esa categoría (H2, H2a, H2b y H2c).

Tabla 10

Prueba de diferencias de medias niveles producto y marca

\begin{tabular}{|c|c|c|c|c|c|}
\hline Dimensión & Reactivos & $\begin{array}{l}\text { Producto: } \\
\text { Café }\end{array}$ & $\begin{array}{l}\text { Marca: } \\
\text { Starbucks }\end{array}$ & & \\
\hline & & Media & Media & $\mathrm{t}$ & $\mathrm{Z}$ \\
\hline \multirow{6}{*}{$\begin{array}{l}\text { Atención } \\
\text { Consciente }\end{array}$} & Me gusta aprender sobre & 3.72 & 2.05 & $8.3^{* *}$ & $-5.565 * *$ \\
\hline & Pongo mucha atención a todo lo relacionado con & & & & $-5.064 * *$ \\
\hline & & 3.23 & 1.87 & $6.6^{* *}$ & \\
\hline & Me mantengo al tanto de novedades relacionadas & & & & $-3.967 * *$ \\
\hline & con & 2.62 & 1.80 & $4.6^{* *}$ & \\
\hline & capta mi atención a menudo. & 3.18 & 2.10 & $5.1^{* *}$ & $-4.278 * *$ \\
\hline \multirow{6}{*}{$\begin{array}{l}\text { Participación } \\
\text { entusiasta }\end{array}$} & Dedico mucho de mi tiempo personal a & & & & $-4.231 * *$ \\
\hline & & 2.55 & 1.55 & $5.0^{* *}$ & \\
\hline & Mis días no serían iguales sin & 3.22 & 1.32 & $8.3^{* *}$ & $-5.555 * *$ \\
\hline & __ es una parte importante en mi vida. & 3.17 & 1.28 & $8.6^{* *}$ & $-5.492 * *$ \\
\hline & Busco hacer espacios en mi agenda para dedicar & & & & $-4.765 * *$ \\
\hline & $\mathrm{a}$ & 2.63 & 1.33 & $6.3^{* *}$ & \\
\hline \multirow{6}{*}{$\begin{array}{l}\text { Conexión } \\
\text { social }\end{array}$} & Disfruto más ___ cuando estoy & & & & $-4.644 * *$ \\
\hline & acompañado. & 3.55 & 2.27 & $5.9^{* *}$ & \\
\hline & $\begin{array}{l}\text { es más divertido cuando hay más } \\
\text { gente conmigo que también lo hace. }\end{array}$ & 3.42 & 2.00 & $6.4^{* *}$ & $-4.799 * *$ \\
\hline & $\ldots$ es algo que comparto con mis & & & & $-5.013 * *$ \\
\hline & amigos. & 3.17 & 1.85 & $6.6^{* *}$ & \\
\hline & $\begin{array}{l}\text { es una buena manera de convivir } \\
\text { con familia y amigos. }\end{array}$ & 3.67 & 1.93 & $7.7^{* *}$ & $-5.303 * *$ \\
\hline
\end{tabular}

$\mathrm{n}=60$ Prueba de comparación de medias pareadas, **Prueba t con nivel de significancia a .01 o menor.

$\mathrm{t}=$ prueba $\mathrm{t}$ de diferencia de medias. $\mathrm{Z}=$ prueba de diferencia de rangos de Wilcoxon.

Fuente: elaboración propia. 
Tabla 11

Análisis factorial confirmatorio. Separación entre engagement hacia el producto y hacia la marca

Componente

\begin{tabular}{|c|c|c|}
\hline & 1 & 2 \\
\hline Pongo mucha atención a todo lo relacionado con Starbucks. & 0.883 & \\
\hline Dedico mucho de mi tiempo personal a Starbucks. & 0.863 & \\
\hline Mis días no serían iguales sin ir a Starbucks. & 0.837 & \\
\hline Starbucks es una parte importante en mi vida. & 0.825 & \\
\hline Starbucks capta mi atención a menudo. & 0.821 & \\
\hline Me gusta aprender sobre Starbucks. & 0.814 & \\
\hline Me mantengo al tanto de las novedades relacionadas con Starbucks. & 0.807 & \\
\hline Starbucks es una buena manera de convivir con familia y amigos. & 0.802 & \\
\hline Busco hacer espacios en mi agenda para dedicar a Starbucks. & 0.789 & \\
\hline Starbucks es más divertido cuando estoy con gente que también le gusta Starbucks. & 0.747 & \\
\hline Starbucks es algo que comparto con mis amigos. & 0.712 & \\
\hline Disfruto más Starbucks cuando estoy acompañado. & 0.586 & \\
\hline El café capta mi atención a menudo. & & 0.836 \\
\hline Pongo mucha atención a todo lo relacionado con el café. & & 0.815 \\
\hline Me mantengo al tanto de las novedades relacionadas con el café. & & 0.809 \\
\hline Dedico mucho de mi tiempo personal a tomar café. & & 0.806 \\
\hline Tomar café es una parte importante en mi vida. & & 0.801 \\
\hline Busco hacer espacios en mi agenda para dedicar a tomar café. & & 0.745 \\
\hline Tomar café es algo que comparto con mis amigos. & & 0.734 \\
\hline Me gusta aprender sobre café. & & 0.723 \\
\hline Mis días no serían iguales sin tomar café. & & 0.703 \\
\hline Tomar café es una buena manera de convivir con familia y amigos. & & 0.621 \\
\hline Tomar café es más divertido cuando estoy con gente que también lo hace. & & 0.573 \\
\hline Disfruto más tomar café cuando estoy acompañado. & & 0.540 \\
\hline
\end{tabular}

Nota: Análisis factorial confirmatorio con rotación varimax. Se omiten cargas por debajo de 0.5

Fuente: elaboración propia

\section{Conclusiones}

La distinción del engagement hacia un producto y hacia una marca podría ser una herramienta valiosa para el establecimiento de estrategias de mercadotecnia efectivas. Si bien es necesario analizar más profundamente las diferencias entre los niveles de las tres dimensiones y entre ambos niveles de análisis, el poder identificar que existe una diferencia es un primer paso 
hacia un entendimiento más profundo de este concepto. Al tener este entendimiento de las diferencias, podría contarse con un instrumento útil de comparación entre distintos oferentes en una misma categoría de producto ya que un engagement más elevado podría ser el reflejo de un valor agregado que es percibido más claramente por el consumidor. Como los resultados indican, en este caso se encontró que el nivel de engagement en cada una de las dimensiones es superior hacia la marca (Starbucks) utilizada en este estudio que hacia la categoría del producto (café en taza). Esto permite sugerir que la marca puede ser un elemento que puede transferir valor hacia la categoría de producto amparada al permitir un incremento en el nivel total de engagement en el proceso de elección de compra.

Por otro lado, el engagement de una categoría podría ser un indicador importante de referencia y distinción para las marcas que logran superar ese nivel y aquellas que no logran alcanzarlo. Investigaciones futuras podrían incluir esta relación entre valor percibido y engagement, así como el impacto que generan distintas acciones de comunicación, innovación, educación al consumidor, etc. en este indicador. Se ha remarcado la necesidad de estudios futuros dirigidos hacia la comprensión de las diferencias de engagement hacia diferentes objetos focales (i.e. categoría de producto, marca) y sus implicaciones (Dessart et al., 2016). El presente estudio presenta una primera aproximación a la distinción del engagement hacia distintos objetos, particularmente entre aquellos que tendrían relevancia para estrategias de branding (el término en inglés es aceptado en la literatura como el proceso de creación y desarrollo de valor de marca) y comunicación. En este caso, se ha ofrecido evidencia de la diferencia entre el nivel de engagement hacia una categoría de producto y aquel hacia una marca en particular dentro de tal categoría.

En este estudio se han presentado evidencias para ofrecer un instrumento que puede servir para medir el grado de engagement de un consumidor hacia algún objeto. Las pruebas de confiabilidad y validez reportadas en general tienden a sugerir que los reactivos propuestos son útiles como indicadores de sus dimensiones en un contexto mexicano. Adicionalmente, con base en los resultados obtenidos, se encontró que el engagement puede ser distinto para distintos niveles de objetos en marketing (nivel categoría de producto y nivel marca). A través de la generación de un instrumento que ha dado ciertas evidencias de ser válido y confiable para el contexto mexicano, se presenta la posibilidad de poder continuar la línea de investigación sobre engagement. Futuros estudios podrían incluir el desarrollar la capacidad de generalización del instrumento hacia otras categorías de producto. Habiendo definido la validez predictiva de la escala, podrían explorarse modelos para un análisis más profundo de los antecedentes y consecuencias del engagement. Adicionalmente, la exploración de niveles distintos de engagement entre distintos tipos de consumidores podría ser la base para identificar una segmentación de mercado alternativa.

La investigación del engagement en categorías de servicios podría requerir una reevaluación y adaptación del instrumento que constituiría también un enfoque relevante para el 


\section{J. Vera-Martínez y S. Ornelas-Sánchez / Contaduría y Administración 66(3), 2021, 1-23 \\ http://dx.doi.org/10.22201/fca.24488410e.2021.1898}

ámbito del marketing. Las limitaciones de la adaptación de la escala incluyen la particularidad de la categoría de producto utilizada (café en taza). Una limitación adicional radica en que la muestra pertenece a consumidores de zonas urbanas del centro de México, lo cual podría también reflejar cierto sesgo. Futuros estudios podrían incluir muestras segmentadas con distintos perfiles de preferencias, zonas geográficas, hábitos de consumo, conocimiento de la categoría y/o consumo específico de ciertas marcas. Adicionalmente, el engagement hacia el café pudiera diferir dado dos mercados distintos: por un lado, tratarse de un producto tradicional en un mercado de consumo masivo, pero por otro, tratarse de un producto en un mercado con una tendencia reciente a la sofisticación (Carvalho et al., 2016).

\section{Referencias}

Baldus, B. J., Voorhees, C., \& Calantone, R. (2015). Online brand community engagement : Scale development and validation. Journal of Business Research, 68(5), 978-985. https://doi.org/10.1016/j.jbusres.2014.09.035

Balsano, A. B. (2005). Youth Civic Engagement in the United States: Understanding and Addressing the Impact of Social Impediments on Positive Youth and Community Development. Applied Development Science, 9(4), 202-215. https://doi.org/10.1207/s1532480xads0904

Bowden, J. L.-H. (2009). The Process of Customer Engagement: A Conceptual Framework. The Journal of Marketing Theory and Practice, 17(1), 63-74. https://doi.org/10.2753/MTP1069-6679170105

Brodie, R. J., \& Hollebeek, L. D. (2011). Response: Advancing and Consolidating Knowledge About Customer Engagement. Journal of Service Research, 14(3), 283-284. https://doi.org/10.1177/1094670511415523

Brodie, R. J., Hollebeek, L. D., Jurić, B., \& Ilić, A. (2011). Customer Engagement. Journal of Service Research, 14(3), 252-271. https://doi.org/10.1177/1094670511411703

Calder, B. J., Malthouse, E. C., \& Schaedel, U. (2009). An Experimental Study of the Relationship between Online Engagement and Advertising Effectiveness. Journal of Interactive Marketing, 23(4), 321-331. https://doi. org/10.1016/j.intmar.2009.07.002

Carvalho, J. M., Paiva, E. L., \& Vieira, L. M. (2016). Quality attributes of a high specification product. British Food Journal, 118(1), 132-149. https://doi.org/10.1108/BFJ-02-2015-0059

Catteeuw, F., Flynn, E., \& Vonderhorst, J. (2007). Employee engagement : Boosting productivity in turbulent times. Organization Development Journal, 25(2), 151-157. https://doi.org/10.1017/CBO9781107415324.004

Dabholkar, P. A. (1990). How to Improve Perceived Service Quality by Increasing Customer Participation. Developments in Marketing Science: Proceedings of the Academy of Marketing Science, 483-487. https://doi. org/10.1007/978-3-319-13254-9_97

Dessart, L., Veloutsou, C., \& Morgan-Thomas, A. (2016). Capturing consumer engagement: duality, dimensionality and measurement. Journal of Marketing Management, 32(5-6), 399-426. https://doi.org/10.1080/0267257X.2015.1130738

Fischer, E. F., \& Victor, B. (2014). High-end coffee and smallholding growers in Guatemala. Latin American Research Review, 49(1), 155-177. https://doi.org/10.1353/lar.2014.0001

Gambetti, R. C., Graffigna, G., \& Biraghi, S. (2012). The Grounded Theory approach to consumer--brand engagement: the practitioner's standpoint. International Journal of Market Research, 54(5), 659. https://doi. org/10.2501/IJMR-54-5-659-687

Gliem, J. A., \& Gliem, R. R. (2003). Calculating, interpreting, and reporting Cronbach's alpha reliability coefficient for Likert-type scales. Midwest Research to Practice Conference in Adult, Continuing, and Community Education, (1992), 82-88. https://doi.org/10.1109/PROC.1975.9792

Guthrie, J. T., \& Cox, K. E. (2001). Classroom conditions for motivation and engagement in reading. Educational 


\section{J. Vera-Martínez y S. Ornelas-Sánchez / Contaduría y Administración 66(3), 2021, 1-23 \\ http://dx.doi.org/10.22201/fca.24488410e.2021.1898}

psychology review, 13(3), 283-302. https://link.springer.com/article/10.1023/A:1016627907001

Haven, B. (2007). Marketing's New Key Metric: Engagement.

Hernandez, G. (2016). Comunicación personal. Centro Agroecológico del Café A.C., México.

Higgins, E. T., \& Scholer, A. A. (2009). Engaging the consumer: The science and art of the value creation process. Journal of Consumer Psychology, 19(2), 100-114. https://doi.org/10.1016/j.jcps.2009.02.002

Hollebeek, L. (2011). Exploring customer brand engagement: Definition and themes. Journal of Strategic Marketing, 19(7), 555-573. https://doi.org/10.1080/0965254X.2011.599493

Hollebeek, L. D. (2011). Demystifying customer brand engagement: Exploring the loyalty nexus. Journal of Marketing Management, 27(7-8), 785-807. https://doi.org/10.1080/0267257X.2010.500132

Hollebeek, L. L. D., Glynn, M. M. S., \& Brodie, R. J. R. (2014). Consumer brand engagement in social media: Conceptualization, scale development and validation. Journal of Interactive Marketing, 28(2), 149-165. https:// doi.org/10.1016/j.intmar.2013.12.002

Johanson, G. A., \& Brooks, G. P. (2010). Initial Scale Development: Sample Size for Pilot Studies. Educational and Psychological Measurement, 70(3), 394-400. https://doi.org/10.1177/0013164409355692

Jones, E. (2016). Consumer Preferences for Coffee: Hot and Wet, or Quality and Flavor? Journal of Food Products Marketing, 22(3), 350-380. https://doi.org/10.1080/10454446.2014.949973

Lusch, R. F., \& Vargo, S. L. (2006). Service-dominant logic: reactions, reflections and refinements. Marketing Theory, 6(3), 281-288. https://doi.org/10.1177/1470593106066781

Mollen, A., \& Wilson, H. (2010). Engagement, Telepresence and Interactivity in Online Consumer Experience. Journal of Business Research, 63(9 Special Issue on Internet Customer Behavior), 919-925. https://doi.or$\mathrm{g} / 10.1016 / \mathrm{j} . j b u s r e s .2009 .05 .014$

Morales Hernández, J. I., Mendez, M. L., Nolasco Ruíz, S. P., \& Cerón López, M. T. (2018). Proposal for the Creation of a Network of Family Businesses in the MExican Coffee Industry. International Journal of Advanced Engineering Management and Science, 4(11), 773-781. https://doi.org/10.22161/ijaems.4.11.6

Norman, G. (2010). Likert scales, levels of measurement and the "laws" of statistics. Advances in Health Sciences Education, 15(5), 625-632. https://doi.org/10.1007/s10459-010-9222-y

Oliver, R. (1999). Whence Consumer Loyalty? Journal of Marketing, 63(Special Issue), 33-44. https://doi. org/10.2307/1252099

Pansari, A., \& Kumar, V. (2017). Customer engagement: the construct, antecedents, and consequences. Journal of the Academy of Marketing Science, 45(3), 294-311. https://doi.org/10.1007/s11747-016-0485-6

So, K. K. F., King, C., \& Sparks, B. (2014). Customer Engagement With Tourism Brands: Scale Development and Validation. Journal of Hospitality and Tourism Research, 38(3), 304-329. https://doi. org/10.1177/1096348012451456

Sprott, D., Czellar, S., \& Spangenberg, E. (2009). The Importance of a General Measure of Brand Engagement on Market Behavior: Development and Validation of a Scale. Journal of Marketing Research, 46(1), 92-104. https://doi.org/10.1509/jmkr.46.1.92

Van Doorn, J., Lemon, K. N., Mittal, V., Nass, S., Pick, D., Pirner, P., \& Verhoef, P. C. (2010). Customer Engagement Behavior: Theoretical Foundations and Research Directions. Journal of Service Research, 13(3), 253-266. https://doi.org/10.1177/1094670510375599

Verhoef, P. C., Reinartz, W. J., \& Krafft, M. (2010). Customer Engagement as a New Perspective in Customer Management. Journal of Service Research, 13(3), 247-252. https://doi.org/10.1177/1094670510375461

Vivek, S. D., Beatty, S. E., Dalela, V., \& Morgan, R. M. (2014). A Generalized Multidimensional Scale for Measuring Customer Engagement. The Journal of Marketing Theory and Practice, 22(4), 401-420. https://doi. org/10.2753/MTP1069-6679220404

Vivek, S. D., Beatty, S. E., \& Morgan, R. M. (2012). Customer Engagement: Exploring Customer Relationships Beyond Purchase. The Journal of Marketing Theory and Practice, 20(2), 122-146. https://doi.org/10.2753/ MTP1069-6679200201

Zaichkowsky, J. L. (1985). Measuring the Involvement Construct. Journal of Consumer Research. https://doi. org/10.1086/208520 